\title{
GUEST EDITOR'S NOTE: SPECIAL SERIES ON GLOBAL DRUG DEVELOPMENT ISSUES
}

\author{
Kenneth I. Kaitin, PhD \\ Director, Tufts Center for the Study of Drug Development, Tufts University, Boston, Massachusetts
}

OVER THE PAST 30 years, the time taken to bring a new drug to market has increased substantially. Despite recent reductions in regulatory review times, clinical drug development remains a time-consuming, risky, and expensive process. To succeed in today's highly competitive marketplace, the research-based pharmaceutical and biotechnology industries are faced with the daunting challenge of reducing lengthy discovery and development times, increasing the number of products in research and development (R\&D) pipelines, reducing late-stage attrition rates, lowering R\&D costs, and developing breakthrough drugs. The ability of firms to meet this challenge will significantly affect not just those within industry, but also patients, whose demand for newer and more effective drugs grows steadily.

To explore some of the issues that impact on global drug development, a call went out to the Drug Information Association membership to contribute papers for a special series of articles to appear in this issue of the Drug Information Journal. The result is the five papers that follow.

The articles included in the series cover a very broad range of topics. The paper by Klaus Olejniczak, Peter Gunzel, and Rolf Bass on preclinical testing strategies provides a series of instructive flowcharts to

Reprint address: Kenneth I. Kaitin, PhD, Director, Tufts Center for the Study of Drug Development, Tufts University, 192 South St., Suite 550, Boston, MA 02111. E-mail: kkaitin@infonet.tufts.edu. assist in the development of a preclinical strategy that will maximize information flow from preclinical to clinical investigators, as well as from sponsors to regulatory authorities. Janice Reichert compares and contrasts key clinical development benchmarks for biopharmaceutical versus pharmaceutical products. Stephen Klincewicz, John Clark, and Norman LaFrance examine several significant regulations and guidelines that deal with the safety sections of the investigator's brochure. They also explore the utility of comprehensive investigational risk assessments by which manufacturers can meet safetyrelated regulatory obligations. Yvonne Legras provides a summary of important deficiencies identified by European regulatory agencies in the chemical, pharmaceutical, and biological documentation of new drug submissions. She also comments on the presentation of the pharmaceutical dossier and expert report. Finally, Craig McCarthy presents an overview of what has been achieved so far by the International Conference on Harmonization, and explores several missed opportunities.

I wish to thank the authors for sharing their insights and experiences and for taking the time to prepare the articles presented here. I also offer my gratitude and appreciation to Tom Teal, Editor-in-Chief of the Drug Information Journal, for his invaluable assistance in pulling this series together. I trust that you will find the endeavor worthwhile. 